\title{
Gasolinas a partir de metanol (MTG): Intensificación del proceso en un reactor de lecho fluidizado de doble zona (TZFBR)
}

\author{
Andrés Sanz, Javier Lasobras, Jaime Soler, Javier Herguido, Miguel Menéndez \\ Grupo de Catálisis, Separaciones Moleculares e Ingeniería del Reactor (CREG) \\ Instituto de Investigación en Ingeniería de Aragón (I3A) \\ Universidad de Zaragoza, Mariano Esquillor s/n, 50018, Zaragoza, Spain. \\ Tel. +34-976762707, e-mail: sanza@unizar.es
}

\section{Resumen}

Se ha estudiado la viabilidad de producir gasolinas a partir de metanol (proceso MTG) en un reactor alternativo: el de lecho fluidizado de doble zona (TZFBR) [1]. Para ello se han probado tres catalizadores diferentes, basados todos ellos en zeolita HZSM-5 como especie activa.

\section{Introducción}

Los combustibles líquidos, especialmente la gasolina, constituyen la principal fuente de energía para el transporte de personas y mercancías. Si bien se prevé que su demanda mundial aumente en muchas regiones del mundo, su uso conlleva una clara problemática medioambiental $\mathrm{y}$, además, las reservas de crudo están en declive [2], por lo que resulta interesante disponer de suministros alternativos. Con esta base, el proceso MTG (methanol to gasoline) se presenta como una tecnología eficiente en la producción de gasolina de alto octanaje y con opción de aprovechar fuentes renovables.

Este proceso, surgido de manera accidental a comienzos de la década de los 70 en la empresa ExxonMobil, supone la transformación de una corriente de metanol en gasolinas cuando éste se pone en contacto con una zeolita de tipo ZSM-5, la cual resulta adecuada por su elevada superficie específica, acidez y estructura bien definida. La alta concentración de aromáticos y la naturaleza no lineal de las parafinas dan al producto final una elevada calidad como gasolina; teniendo como mayor diferencia con la obtenida del crudo, la presenta de una concentración significativa de dureno $(1,2,4,5$ tetrametilbenceno). Por su elevado punto de fusión $\left(80^{\circ} \mathrm{C}\right)$, y para evitar problemas de fluidez en la gasolina, ExxonMobil determinó un valor límite del $2 \%$ wt. en la gasolina final para su uso en automóviles.

La hipótesis de partida en la que se apoya este trabajo es que la desactivación del catalizador por formación de coque -que actualmente supone un problema operacional-, podrá ser contrarrestada por combustión in situ en el mismo reactor en el que se produce la reacción principal, alcanzadose así un funcionamiento estable y continuo.

\section{Experimental}

Los tres catalizadores han sido preparados por algomeración de la zeolita HZSM-5 $\left(\mathrm{SiO}_{2} / \mathrm{Al}_{2} \mathrm{O}_{3}=30\right)$ con boehmita ('HZ_Boeh') o bentonita ('HZ_Bent' y ' $H Z \_B e n t+I I$ ') y alúmina.

La superficie específica y estructura porosa de los tres catalizadores sintetizados, así como de sus constituyentes por separado (zeolita HZSM-5, boehmita, bentonita y alúmina), fue determinada por adsorción física de $\mathrm{N}_{2}$ (Tristar $3000 \mathrm{~V} 6.08$ de Micromeritics). Por su parte, la correcta preparación del catalizador, así como la eficacia del tratamiento de intercambio iónico se comprobó mediante XRF (Thermo Electron ARL ADVANT/XP con tubo de rayos X de rodio) y XRD (difractómetro Panalytical Empyrean con radiación $\mathrm{CuK}_{\alpha} \lambda=1,5418 \AA$ en el rango $2 \theta$ de $0-90^{\circ}$ ).

Se ha trabajado con dos configuraciones de reactor de lecho fluidizado: el convencional (FBR) y el de doble zona (TZFBR). En el primero se ha llevado a cabo el screening de catalizadores (con posterior elección del más adecuado) y un estudio del efecto de la temperatura $\left(400-500^{\circ} \mathrm{C}\right)$. El segundo se ha utilizado para la validación experimental de la reacción MTG en esta novedosa configuración de reactor. El tiempo espacial se mantuvo constante, correspondiendo a $\mathrm{W} / \mathrm{F}_{\mathrm{A} 0}=11,3 \mathrm{~g} \mathrm{~g}_{\mathrm{cat}} \cdot \mathrm{h} / \mathrm{mol}_{\text {metanol, así }}$ como el trabajo a presión atmosférica. La masa de catalizador usada fue de $15 \mathrm{~g}$ en FBR y $22,5 \mathrm{~g}$ en TZFBR. En los ensayos realizados en configuración FBR, tras la etapa de reacción fue necesario llevar a cabo la regeneración del catalizador por combustión de coque. Todas las regeneraciones se realizaron a $550^{\circ} \mathrm{C}$ con un caudal total de $250 \mathrm{~mL}(\mathrm{STP}) / \mathrm{min}$ ( $2 \%$ vol. $\mathrm{O}_{2}$; balance $\mathrm{N}_{2}$ ). 


\section{Resultados y discusión}

Los tres catalizadores presentan superficies específicas elevadas $-211 \mathrm{~m}^{2} \cdot \mathrm{g}^{-1}$ en el menor de los casos con el catalizador de bentonita sin intercambio iónico- (Tabla 1). El tamaño de microporos $\left(\mathrm{d}_{\mu \mathrm{p}}\right) \mathrm{de}$ los distintos catalizadores es similar y coincide con el de la zeolita inicial.

De manera genérica para todos los experimentos, el rendimiento total a hidrocarburos ligeros (fracción de no condensables) es siempre mayor que a hidrocarburos líquidos (fracción de condensables). Las olefinas ligeras (etileno y propileno) representan la mayor contribución en ligeros, mientras que en líquidos lo hacen los productos de naturaleza aromática (mayoritariamente xilenos). El dureno se mantiene siempre en valores de rendimiento por debajo del límite permisible del $2 \%$ wt. Atendiendo al rendimiento a coque, éste se mantiene siempre en valores muy bajos (en ningún caso llega a representar más del $1 \%$ wt. del carbono total alimentado). La conversión de metanol es en todos los casos superior al $99,9 \%$.

Como catalizador más adecuado se puede seleccionar el que contiene boehmita como aglomerante: mayor ratio de rendimiento a líquidos frente a gases, ausencia de problemas fluidodinámicos y menor selectividad a dureno. Un aumento de temperatura se traduce en un mayor rendimiento a hidrocarburos ligeros y a coque y menor rendimiento a gasolinas. Del estudio de estabilidad se concluye que, para temperaturas altas, el rendimiento a BTX (Benceno + Tolueno $+\mathrm{p}$-Xileno) disminuye progresivamente mientras que aumenta el rendimiento a olefinas ligeras. Este comportamiento se puede considerar como un indicador de inestabilidad del proceso [3].

El proceso MTG en TZFBR con el catalizador HZ_Boeh, proporciona rendimientos a las distintas fases (no condensables, condensables y coque) similares a los obtenidos inicialmente con la configuración FBR convencional. Con respecto a la evolución temporal de rendimientos, los resultados con el TZFBR indican una mayor estabilidad del proceso: (i) el rendimiento a BTX se mantiene constante ( $8 \%$ wt.) en lugar de descender como en FBR, y (ii) no solo se corrige la tendencia a aumentar el rendimiento a olefinas ligeras, sino que se aprecia una clara disminución de éste con el tiempo (Fig. 1).

\section{Conclusiones}

Se ha puesto a punto un proceso nuevo (MTG en reactor TZFBR con catalizadores basados en zeolita
HZSM-5), con su correspondiente instalación experimental y sistemas de análisis y tratamientos de datos. Los resultados son satisfactorios ya que, con la configuración TZFBR, se consigue un importante efecto positivo sobre la estabilidad del proceso, tal como se pretendía.

\section{Referencias}

[1]. HERGUIDO, J. and MENÉNDEZ, M. Advances and trends in two-zone fluidized-bed reactors. Current Opinion in Chemical engineering. 2017, 17, 15-21.

[2]. OWEN, N.A., INDERWILDI, O.R. and KING, D.A. The status of conventional world oil reserves-Hype or cause for concern? Energy Policy. 2010, 38(8), 47434749

[3]. AguAyo, A.T., GAYUBO, A.G., ORTEGA, J.M., OLAZAR, M. and BILBAO, J. Catalyst deactivation by coking in the MTG process in fixed and fluidized bed reactors. Catalysis Today. 1997, 37(3), 239-248.

Tabla 1. Propiedades texturales de los sólidos empleados.

\begin{tabular}{|c|c|c|c|c|}
\hline \multirow[b]{3}{*}{ Muestra } & \multicolumn{2}{|c|}{$\begin{array}{c}\text { Sup. } \\
\text { Específica }\end{array}$} & \multicolumn{2}{|c|}{ Prop. $\mu$ porosas } \\
\hline & $\mathbf{S}_{\text {BET }}{ }^{a}$ & $\mathbf{S}_{\mu \mathbf{p}}{ }^{b}$ & $\mathbf{V}_{\mu \mathbf{p}} \mathbf{b}^{\prime}$ & $d_{\mu p}{ }^{c}$ \\
\hline & \multicolumn{2}{|c|}{$\left[\mathrm{m}^{2} / \mathrm{g}\right]$} & {$\left[\mathrm{cm}^{3} / \mathrm{g}\right]$} & [nm] \\
\hline HZSM-5 & 320 & 202 & 0,11 & 2,2 \\
\hline Boehmita & 212 & - & - & - \\
\hline Bentonita & 23 & 4 & 0,002 & - \\
\hline$\alpha$-Alúmina & 180 & - & - & - \\
\hline HZ_Boeh & 288 & 113 & 0,06 & 2,1 \\
\hline$H Z \_B e n t$ & 211 & 58 & 0,03 & 2,1 \\
\hline$H Z \_B e n t+I I$ & 218 & 57 & 0,03 & 2,0 \\
\hline
\end{tabular}

${ }^{\text {a }}$ Método BET; ${ }^{\text {b }}$ Método t-plot $(\delta \sim 0,354-0,5) ;{ }^{\mathrm{c}}$ Método 4V/A

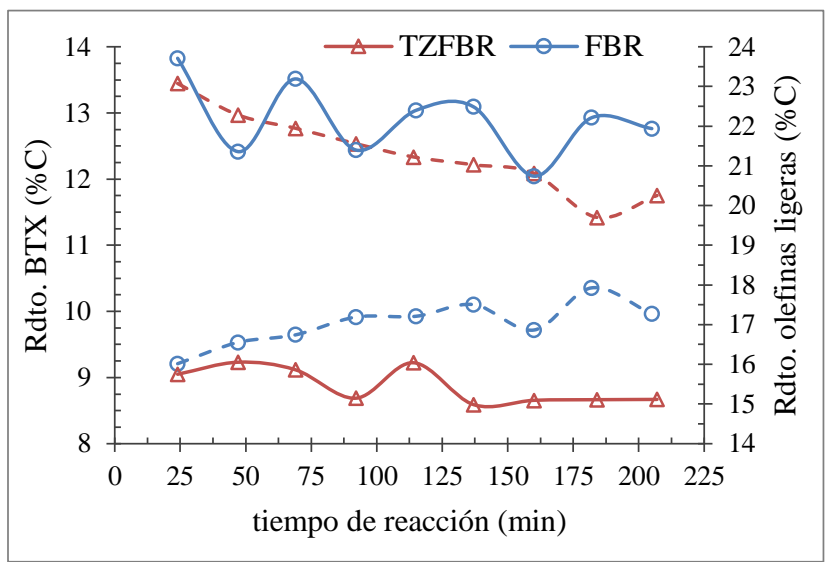

Figura 1. Evolución temporal del rendimiento a BTX -línea continua- y a olefinas ligeras -línea discontinua-. Catalizador $H Z \_B o e h\left[450^{\circ} \mathrm{C} ; \mathrm{W} / \mathrm{F}_{\mathrm{A} 0}=11,3 \mathrm{gcat} \cdot \mathrm{h} / \mathrm{mol}_{\text {metanol }}\right]$. 